\title{
Patient-derived xenograft (PDX) models: an emerging platform for cancer drug development and translational research
}

\begin{abstract}
There is currently a focus on developing and characterizing patient-derived tumor xenograft (PDX) models for cancer research. PDX models are being used for pre-clinical drug evaluation, biomarker identification, biologic studies, and personalized medicine strategies as they mostly retain the principal histologic and genetic characteristics of their donor tumor. Moreover, they remain stable across passages and have proven to be predictive of clinical outcomes. As PDXs grow in popularity, we are developing new techniques to increase their usefulness for patient stratification and treatment design.
\end{abstract}

Volume 3 Issue 4 - 2016

\author{
Joseph F Murphy \\ Charles River Laboratories, USA
}

Correspondence: Joseph F Murphy, Charles River Laboratories, 3300 Gateway Center Blvd, Morrisville, NC 27560, USA., Tel I-9192502318, Email joseph.murphy@crl.com

Received: June 29, 2016 | Published: June 30, 2016
Abbreviations: PDX, patient-derived tumor xenograft; SCID, severely compromised immuno-deficient; TMA, tissue microarray

\section{Editorial}

Previously, cancer drug development was an arena that relied almost exclusively on transgenic and severely compromised immunodeficient (SCID) mice to measure tumor growth in response to novel therapeutic drugs. Development has now evolved to the point where several different murine models are now employed. The industry has expanded to include syngeneic and humanized mice, in addition to PDX models. These models have grown more sophisticated and are being employed, both pre-clinically and co-clinically, to improve drug translation. PDX models have emerged as a powerful technology, and now offer a promising platform for translational cancer research as they retain the molecular heterogeneity of their originating tumor. As a result of these developments, the availability of large collections of such models has become necessary to help forecast responses in clinical trials. Furthermore, the molecular characterization of models is crucial for adequate tumor selection prior to drug testing and for predictive biomarker discovery.

One of the main problems associated with pre-clinical models is that they often fail to capture the diverse heterogeneity of human malignancies, and therefore lack clinical predictive power. The tumor microenvironment is governed by both cell-autonomous (e.g. genetic and epigenetic heterogeneity) and non-cell-autonomous (e.g. stromal heterogeneity) modulators. Whereas PDXs can largely recapitulate the poly-genomic architecture of human tumors, they do not fully account for heterogeneity within the tumor microenvironment. Thus, these models are efficacious in basic and translational research in cancer biology, but limited in their ability to study stromal or immune modulators of malignant progression. Moreover, PDX models facilitate conducting patient specific in vivo and ex vivo drug screens; however treatment responses to stromal contributions may be incomplete.
Given this context, Charles River Laboratories has evaluated over 440 PDXs growing in immunocompromised mice that were successfully established from patient tumors with diverse cancer types. At our Oncotest facility, these PDXs have been extensively characterized for histological features, molecular data, and for tumor growth with sensitivity to relevant standard-of-care compounds and targeted therapies. A histology review of stromal content, differentiation and vasculature level, was performed on all PDX models. A tissue microarray (TMA) was prepared as a "compendiumassociated screening tool" for on-demand target investigation. Raw data was analyzed by PDX-specific bioinformatics pipelines, and processed data was integrated into a growing database for tumor selection, data visualization, and analytics. Details outlining the design and execution of these studies are available at: http://www2. criver.com/1/60962/2016-06-17/9bmhg5.

The availability of well-characterized pre-clinical tumor models with histological and molecular data improves selection of appropriate models for in vitro/in vivo drug testing. This is essential to increase the success rate of pre-clinical studies and facilitate predictive biomarker discovery. In addition to their utility as a drug screening model, using PDX tumor models allows for the identification of biomarkers that predict the tumor's response to therapy. Furthermore, the assessment of PDX models on broad screening platforms, such as 3D culture and in vivo mouse clinical trials (humanized and immuno-deficient), accelerates compound evaluation. Utilizing a combination of these technologies may help circumvent some of the hurdles relating to tumor heterogeneity, thus facilitating a more streamlined approach to cancer treatment.

\section{Acknowledgments}

None.

\section{Conflicts of Interset}

None. 